\title{
Disease activity and patient-reported outcomes in patients with rheumatoid arthritis and Sjögren's syndrome enrolled in a large observational US registry
}

\author{
Leslie R. Harrold ${ }^{1,2}$ (1) $\cdot$ Ying Shan $^{1} \cdot$ Sabrina Rebello ${ }^{1}\left(\mathbb{D} \cdot\right.$ Neil Kramer $^{3} \cdot$ Sean E. Connolly ${ }^{4}$ Evo Alemao ${ }^{4}$. \\ Sheila Kelly ${ }^{4} \cdot$ Joel M. Kremer ${ }^{5}$ (D) Elliot D. Rosenstein ${ }^{3}$ (D)
}

Received: 13 March 2020 / Accepted: 5 May 2020 / Published online: 24 May 2020

(c) The Author(s) 2020

\begin{abstract}
The objective of this study was to compare rheumatoid arthritis (RA) disease activity and patient-reported outcomes (PROs) in a national sample of patients with RA with/without Sjögren's syndrome (SS). Adults with RA from a large observational US registry (Corrona RA) with known SS status between 22 April 2010 and 31 July 2018 and a visit $12( \pm 3)$ months after index date were identified ( $n=36,256 / 52,757)$. SS status: determined from a yes/no variable reported at enrolment into the Corrona RA registry and follow-up visits. Index date: date that SS status was recorded (yes/no). Patients received biologic or targeted synthetic disease-modifying antirheumatic drugs as part of standard care. Patients with RA only were followed for $\geq 12$ months to confirm the absence of SS. Patients were frequency- and propensity-score matched (PSM) 1:1 and stratified by disease duration and treatment response-associated variables, respectively. Clinical Disease Activity Index (CDAI) and PROs 12 months after index visit were compared in patients with and without SS. Baseline characteristics in 283 pairs of PSM patients were balanced. Mean change in CDAI score was numerically lower in patients with RA and SS than patients with RA only (8.8 vs 9.3). Reductions in PROs of pain, fatigue and stiffness were two- to threefold lower for patients with RA and SS versus RA only. Reductions in RA disease activity and RA-related PROs were lower in patients with RA and SS versus those with RA only. Our data indicate that SS adds to treatment challenges; physicians may wish to consider SS status when managing patients with RA.
\end{abstract}

Keywords Sjögren's syndrome $\cdot$ Rheumatoid arthritis $\cdot$ Treatment response $\cdot$ Disease activity $\cdot$ Patient-reported outcomes · ACPA

Electronic supplementary material The online version of this article (https://doi.org/10.1007/s00296-020-04602-8) contains supplementary material, which is available to authorized users.

Leslie R. Harrold

lharrold@corrona.org

Ying Shan

yshan@corrona.org

Sabrina Rebello

srebello@corrona.org

Neil Kramer

Neil.Kramer@atlantichealth.org

Sean E. Connolly

Sean.Connolly@bms.com

Evo Alemao

Evo.Alemao@bms.com

Sheila Kelly

sheila.kelly@bms.com
Joel M. Kremer

jkremer@joint-docs.com

Elliot D. Rosenstein

Elliot.Rosenstein@atlantichealth.org

Corrona, LLC, 1440 Main Street, Suite 310, Waltham, MA 02451, USA

2 University of Massachusetts Medical School, Worcester, MA, USA

3 Institute for Rheumatic \& Autoimmune Diseases, Overlook Medical Center, Summit, NJ, USA

4 Bristol Myers Squibb, Princeton, NJ, USA

5 Albany Medical College and the Center for Rheumatology, Albany, NY, USA 


\section{Introduction}

Sjögren's syndrome (SS) is a systemic autoimmune disease that can occur independently or in conjunction with another autoimmune condition, such as rheumatoid arthritis (RA) [1, 2]. SS has traditionally been thought of as either primary (SS only) or secondary; however, the terminology for secondary SS has recently evolved to be more descriptive, particularly because SS and other autoimmune diseases are co-existing conditions, rather than one being secondary to the other [2].

The current American College of Rheumatology (ACR) and European League Against Rheumatism (EULAR) treatment guidelines for RA and the ACR/EULAR classification criteria for SS do not include recommendations for treating patients with both RA and SS [3-5]. However, patients with both RA and SS have an increased disease burden [6, 7] and a decreased quality of life [8] compared with patients with only one autoimmune disease. For example, patients with RA and SS are more likely to have a longer duration of RA and worse joint damage compared with patients with RA only [6, 9]. Patients with SS alone are more likely to experience a negative impact on physical functioning (e.g., lifting and carrying, climbing stairs, bending and walking moderate distances) and social functioning (e.g., quality and quantity of social activities with others) than control subjects without dry eye disease or SS $[8,10]$.

The correlation of SS with increased disease burden in patients with RA includes an association with higher RA disease activity and anti-citrullinated protein antibody (ACPA) positivity $(+)[6,11]$. ACPAs play a pivotal role in the progression of RA [11, 12], indicating a poor prognosis [4], more severe disease course and radiological destruction compared with patients with RA who are ACPA negative (-) [13]. However, there are limited data on the impact of ACPA positivity and SS status in patients with RA and SS [6, 9, 14, 15].

Evaluating real-world data from patients with RA and SS and patients with RA only may enable clinicians to understand the different needs of these populations. The primary objective of this study was to compare RA disease activity and patientreported outcomes (PROs) in patients from a national registry sample of patients with RA, with and without SS. A secondary objective was to compare RA disease activity and PROs in a sub-group of ACPA+ patients with RA, as measured by anticyclic citrullinated peptide (anti-CCP), with and without SS.

\section{Materials and methods}

\section{Data source}

The Consortium of Rheumatology Researchers of North America (Corrona) RA registry is an independent, prospective, national, observational cohort in which standardized and uniform treatment and outcome data are collected from treating rheumatologists at the time of a clinical encounter for treatment of patients with RA. Patients have been recruited from 182 private practices and academic sites across 42 US states, with 781 participating rheumatologists. As of June 2019, the Corrona RA registry included information on 52,757 patients. Data on 397,236 patient visits and approximately 188,161 patient-years of follow-up observation time have been collected, with a mean patient follow-up of 4.5 (median 3.3) years. The characteristics of the Corrona registry have been described previously [16].

This study was carried out in accordance with the Declaration of Helsinki. All participating investigators were required to obtain full institutional review board (IRB) approval for conducting non-interventional research involving human subjects. Sponsor approval and continuing review was obtained through a central IRB (New England Independent Review Board, NEIRB No. 120160610). For academic investigative sites that did not receive a waiver to use the central IRB, full board approval was obtained from the respective governing IRBs and documentation of approval was submitted to Corrona, LLC, prior to initiating any study procedures. All registry patients were required to provide written informed consent and authorization prior to participating.

\section{Study population}

This study included adult patients with rheumatologistdiagnosed RA enrolled in the Corrona RA registry between 22 April 2010 and 31 July 2018 (Fig. 1). SS status was captured by physicians at enrolment and follow-up visits using a provider form that included a yes/no question regarding the status of SS associated with RA. The presence of SS was determined clinically based upon the presence, or absence, of symptomatic dry eyes and/or mouth judged by the treating physician not to be related to medications. The index date was defined as the date the provider first reported SS status as yes (patients with RA and SS) or no (patients with RA only). Data were included if patients had at least one visit assessing SS status (SS associated with RA: yes/no) and at least 12 months of follow-up after index date. If data were available for more than one visit, the visit closest to the 12-month post-index date was used. Both patients with and without SS were required to have initiated a biologic (b) or targeted synthetic (ts) disease-modifying antirheumatic drug (DMARD). Patients without b/tsDMARD initiation or with missing SS information were excluded. 


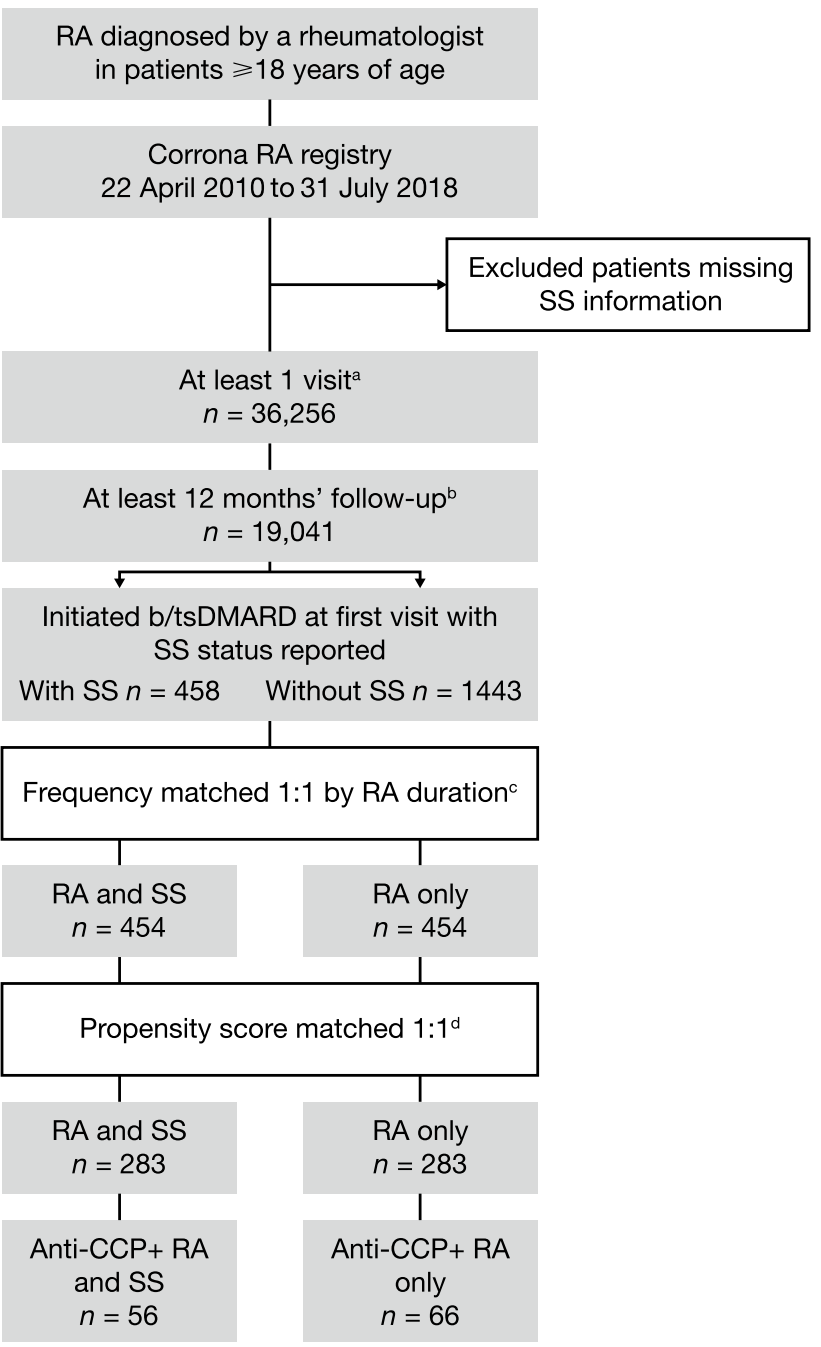

Fig. 1 Selection of eligible patients for analysis. ${ }^{a}$ Yes/no to having SS. ${ }^{b}$ After the first capture of SS data in patients with a diagnosis of no SS. ${ }^{c}$ Between patients with and without SS by biologic initiators. ${ }^{\mathrm{d}}$ Informed from frequency matching, based on logistic regression model predicting group (RA and SS versus RA only). b/tsDMARD biologic or targeted synthetic disease-modifying antirheumatic drug, $C C P+$ cyclic citrullinated peptide positive, $R A$ rheumatoid arthritis, SS Sjögren's syndrome

\section{Study assessments}

Patients were randomly frequency matched 1:1 (patients with RA and SS and patients with RA only) based on the duration of RA $(<2, \geq 2$ to $<5, \geq 5$ to $<10, \geq 10$ to $<15, \geq 15$ to $<20, \geq 20$ to $<30$ and $\geq 30$ years). Due to residual imbalance after frequency matching, frequencymatched patients were pooled together and propensityscore matched (PSM) 1:1 based on a logistic regression model-predicting group (RA and SS versus RA only). In the propensity score model, baseline demographics and response characteristics were compared between the cohorts (RA and SS versus RA only), and the absolute values of the standardized differences were estimated. The absolute value of the standardized difference of $\leq 0.1$ for the overall population [17] was taken to indicate a negligible difference in the mean or prevalence of a covariate between cohorts; variables with a standardized difference $>0.1$ were included in the construction of the propensity score model.

Variables assessed included demographic and socioeconomic characteristics, lifestyle factors, history of comorbidities, RA disease characteristics, previous and current RA therapy, RA disease activity and PROs. Patients with an SS diagnosis (patients with RA and SS) were compared with patients without an SS diagnosis (patients with RA only). For the subgroup analysis in patients who were anti$\mathrm{CCP}+$, anti-CCP testing was performed by investigators and anti-CCP+ was defined as $\geq 20$ units $/ \mathrm{mL}$. Statistical significance was estimated using Student's $t$ test for continuous variables and Chi-square test for category variables.

\section{Study outcomes}

The primary outcome was the mean change in Clinical Disease Activity Index (CDAI) score from index visit to Month 12 in PSM patients [18]. The secondary outcome was mean change in PROs (modified Health Assessment Questionnaire [mHAQ], pain, fatigue, patient global assessment, EuroQol 5 dimensions [EQ-5D] index and morning stiffness) from index visit to Month 12 in PSM patients. Subgroup analyses were conducted in patients who were anti-CCP+ at index visit.

\section{Results}

SS data were available for 36,256 patients with RA, of whom 1901 met the inclusion criteria (Fig. 1). There were 454 pairs of patients randomly generated with either RA and SS or RA only by frequency matching based on RA duration (Fig. 1 and Supplementary Table S1). Due to residual cohort imbalance after frequency matching (Supplementary appendix), the frequency-matched cohorts were pooled and further refined by PSM. Of the 454 pairs of frequency-matched patients, 283 pairs of patients were PSM (Table 1 and Fig. 1). The baseline demographics, disease characteristics and PROs of PSM patients with RA, with and without SS, were generally well balanced. At baseline, $62-64 \%$ of patients were being treated with tumor necrosis factor inhibitors, $21-22 \%$ with other b/tsDMARDs and 16-17\% with abatacept. At baseline, 56-59\% of patients in each cohort were receiving methotrexate monotherapy. 
Table 1 Baseline characteristics of the PSM RA cohort

\begin{tabular}{|c|c|c|c|}
\hline & $\begin{array}{l}\text { Patients with RA and } \\
\text { SS }(n=283)\end{array}$ & $\begin{array}{l}\text { Patients with RA } \\
\text { only }(n=283)\end{array}$ & $p$ value \\
\hline Age, years, mean (SD) & $58.4(12.2)$ & $58.7(12.8)$ & 0.712 \\
\hline Sex, female & $235(83.0)$ & $235(83.0)$ & 1.000 \\
\hline Work status & & & 0.945 \\
\hline Full-time & $103(36.4)$ & $107(37.8)$ & \\
\hline Part-time & $33(11.7)$ & $29(10.2)$ & \\
\hline Disabled & $42(14.8)$ & $46(16.3)$ & \\
\hline Retired & $84(29.7)$ & $83(29.3)$ & \\
\hline Other & $21(7.4)$ & $18(6.4)$ & \\
\hline BMI, kg/m², mean (SD) & $30.2(7.0)$ & $30.5(7.1)$ & 0.699 \\
\hline Duration of RA, years, mean (SD) & $10.5(10.0)$ & $10.3(9.7)$ & 0.834 \\
\hline \multicolumn{4}{|l|}{ Co-morbidities } \\
\hline Hypertension & $89(31.4)$ & $91(32.2)$ & 0.857 \\
\hline CV disease ${ }^{a}$ & $32(11.3)$ & $31(11.0)$ & 0.894 \\
\hline Malignancy ${ }^{\mathrm{b}}$ & $29(10.2)$ & $31(11.0)$ & 0.785 \\
\hline Diabetes & $27(9.5)$ & $25(8.8)$ & 0.771 \\
\hline Serious infections ${ }^{c}$ & $24(8.5)$ & $24(8.5)$ & 1.000 \\
\hline Asthma & $18(6.4)$ & $15(5.3)$ & 0.590 \\
\hline COPD & $5(1.8)$ & $7(2.5)$ & 0.560 \\
\hline ILD/pulmonary fibrosis & $4(1.4)$ & $1(0.4)$ & 0.178 \\
\hline Anti-CCP+, n/m (\%) & $56 / 114(49.1)$ & $66 / 119(55.5)$ & 0.333 \\
\hline $\mathrm{RF}+, \mathrm{n} / \mathrm{m}(\%)$ & $91 / 137(66.4)$ & $80 / 144(55.6)$ & 0.062 \\
\hline Erosive disease, n/m (\%) & $74 / 216(34.3)$ & $64 / 197(32.5)$ & 0.703 \\
\hline Subcutaneous nodules, n/m (\%) & $69 / 283(24.4)$ & $73 / 283(25.8)$ & 0.698 \\
\hline Current b/tsDMARDs use & $283(100.0)$ & $283(100.0)$ & \\
\hline TNFi & $180(63.6)$ & $174(61.5)$ & 0.602 \\
\hline Other b/tsDMARD & $59(20.8)$ & $62(21.9)$ & 0.758 \\
\hline Abatacept & $44(15.5)$ & $47(16.6)$ & 0.731 \\
\hline Concomitant csDMARD use, n/m (\%) & $207 / 283(73.1)$ & $213 / 283(75.3)$ & 0.848 \\
\hline MTX only & $121(58.5)$ & $119(55.9)$ & \\
\hline Non-MTX csDMARD only & $41(19.8)$ & $40(18.8)$ & \\
\hline MTX and non-MTX combination & $39(18.8)$ & $46(21.6)$ & \\
\hline Non-MTX and csDMARD combination & $6(2.9)$ & $8(3.8)$ & \\
\hline Number of prior b/tsDMARDs & & & 0.794 \\
\hline 0 & $116(41.0)$ & $121(42.8)$ & \\
\hline 1 & $77(27.2)$ & $70(24.7)$ & \\
\hline$\geq 2$ & $90(31.8)$ & $92(32.5)$ & \\
\hline Number of prior csDMARDs & & & 0.871 \\
\hline 0 & $16(5.7)$ & $16(5.7)$ & \\
\hline 1 & $116(41.0)$ & $110(38.9)$ & \\
\hline$\geq 2$ & $151(53.4)$ & $157(55.5)$ & \\
\hline CDAI score, mean (SD) & $24.0(14.9)$ & $24.1(14.7)$ & 0.922 \\
\hline \multicolumn{4}{|l|}{ PROs } \\
\hline mHAQ, mean (SD) & $0.5(0.5)$ & $0.6(0.5)$ & 0.389 \\
\hline Patient pain, mean $(\mathrm{SD})^{\mathrm{d}}$ & $50.6(27.0)$ & $52.3(27.3)$ & 0.464 \\
\hline Patient fatigue, mean $(\mathrm{SD})^{\mathrm{e}}$ & $53.6(29.3)$ & $56.2(28.9)$ & 0.299 \\
\hline Patient global assessment, mean (SD) & $47.8(26.1)$ & $50.0(25.4)$ & 0.303 \\
\hline EQ-5D index, mean $(S D)^{f}$ & $0.7(0.2)$ & $0.7(0.2)$ & 0.414 \\
\hline Morning stiffness time, minutes, mean $(\mathrm{SD})^{\mathrm{g}}$ & $111.1(199.1)$ & $112.3(195.5)$ & 0.942 \\
\hline
\end{tabular}

Data are $n(\%)$ unless otherwise stated

${ }^{a}$ History of coronary artery disease, myocardial infarction, congestive heart failure requiring hospitaliza- 
Table 1 (continued)

tion, acute coronary syndrome, unstable angina, cardiac revascularization procedure, cardiac arrest, ventricular arrhythmia, stroke, transient ischemic attack, or other CV event

${ }^{b}$ History of lung cancer, breast cancer, lymphoma, skin cancer (melanoma and squamous cell carcinoma), or other cancer

${ }^{\mathrm{c}}$ Infection required hospitalization or IV treatment

${ }^{\mathrm{d}}$ Patients with RA and SS, $n=282$

ePatients with RA and SS, $n=271$; patients with RA only, $n=237$

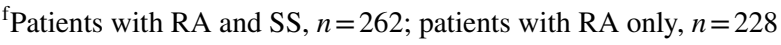

${ }^{g}$ Patients with RA and SS, $n=251$; patients with RA only, $n=247$

$B M I$ body mass index, $b / t s D M A R D$ biologic or targeted synthetic disease-modifying antirheumatic drug, $C C P+$ cyclic citrullinated peptide positive, $C D A I$ Clinical Disease Activity Index; COPD chronic obstructive pulmonary disease, $c s D M A R D$ conventional synthetic disease-modifying antirheumatic drug, $C V$ cardiovascular, $E Q-5 D$, EuroQol 5 dimension, $I L D$ interstitial lung disease, $I V$ intravenous, $m H A Q$ modified Health Assessment Questionnaire, $M T X$ methotrexate, $n / m$ number of patients/total number of patients with available data, $P R O$ patient-reported outcome, $R A$ rheumatoid arthritis, $R F+$ rheumatoid factor positive, $S D$ standard deviation, $S S$ Sjögren's syndrome, TNFi tumor necrosis factor inhibitor

Table 2 Mean CDAI score and PROs at the 12-month follow-up

\begin{tabular}{lccc}
\hline & $\begin{array}{c}\text { Patients with RA } \\
\text { and SS }(n=283)\end{array}$ & $\begin{array}{l}\text { Patients with } \\
\text { RA only } \\
(n=283)\end{array}$ & $p$ value \\
\hline CDAI & $15.2(13.4)$ & $14.8(12.8)$ & 0.758 \\
mHAQ & $0.5(0.5)$ & $0.5(0.5)$ & 0.629 \\
Patient pain & $44.2(29.0)$ & $38.3(28.1)$ & 0.014 \\
Patient fatigue & $49.2(30.1)$ & $43.4(28.5)$ & 0.018 \\
$\begin{array}{l}\text { Patient global assess- } \\
\quad \text { ment }\end{array}$ & $42.4(27.8)$ & $37.1(27.4)$ & 0.023 \\
$\begin{array}{l}\text { EQ-5D index } \\
\text { Morning stiffness }\end{array}$ & $0.7(0.2)$ & $0.7(0.2)$ & 0.982 \\
$\quad$ (min) & $92.9(172.7)$ & $90.6(209.0)$ & 0.922 \\
\hline
\end{tabular}

Data are mean (SD)

$C D A I$ Clinical Disease Activity Index, $E Q-5 D$ EuroQol 5-dimension, $m H A Q$ modified Health Assessment Questionnaire, PRO patientreported outcome, $R A$ rheumatoid arthritis, $S D$ standard deviation, $S S$ Sjögren's syndrome

\section{RA disease activity}

At the 12-month follow-up, mean (standard deviation [SD]) CDAI score was numerically higher in patients with RA and SS compared with patients with RA only (Table 2). At the 12-month follow-up, the mean (SD) change in CDAI score from the index visit was numerically lower in patients with RA and SS compared to those with RA only (Fig. 2). These comparisons did not reach statistical significance.

\section{Patient-reported outcomes}

At baseline, patients with RA and SS had lower mean (SD) PRO scores than patients with RA only (Table 1). However, at the 12-month follow-up visit, mean (SD) PRO scores were higher in patients with RA and SS compared with patients with RA only (Table 2), with the exception of mHAQ and EQ-5D index scores, which were similar in both cohorts. Mean (SD) patient pain, patient fatigue, and patient global assessment at the 12-month follow-up visit were significantly higher in patients with RA and SS compared with patients with RA only ( $p=0.014, p=0.018$ and $p=0.023$, respectively). Morning stiffness at 12 months was numerically higher in patients with RA and SS compared with patients with RA only, but this was not statistically significant. Mean changes from the index visit in pain, fatigue and patient global assessment were statistically significant ( $p=0.002, p=0.001$ and $p=0.002$, respectively), and twoto three-fold inferior (suggesting more impairment), for patients with RA and SS compared with patients with RA only (Fig. 2). The mean change in morning stiffness was numerically lower in patients with RA and SS compared with patients with RA only. The mean change in mHAQ was similar in both cohorts. There was no change in EQ-5D index score from index event until 12-month follow-up for patients with RA and SS, while the mean change for patients with RA only was marginally worse at 12 months.

\section{Subgroup analyses in patients with anti-CCP+RA}

Of the 283 PSM pairs, there were 122 patients who were anti-CCP+: 56 (45.9\%) patients with RA and SS and 66 (54.1\%) patients with RA only (Fig. 1). In patients who were anti-CCP+, the mean (SD) change in CDAI score was similar between patients with RA and SS and those with RA only (Fig. 3). In patients who were anti-CCP+, the mean (SD) change in pain and fatigue was significantly lower in patients with RA and SS compared with patients with RA only ( $p=0.048$ and $p=0.003$, respectively; Fig. 3). Although numerical differences were observed, there were no statistically significant differences in mean improvements in patient global assessment and morning stiffness outcomes between patients with anti-CCP+RA and SS and those with anti-CCP+RA only. In patients who were anti-CCP+, the 


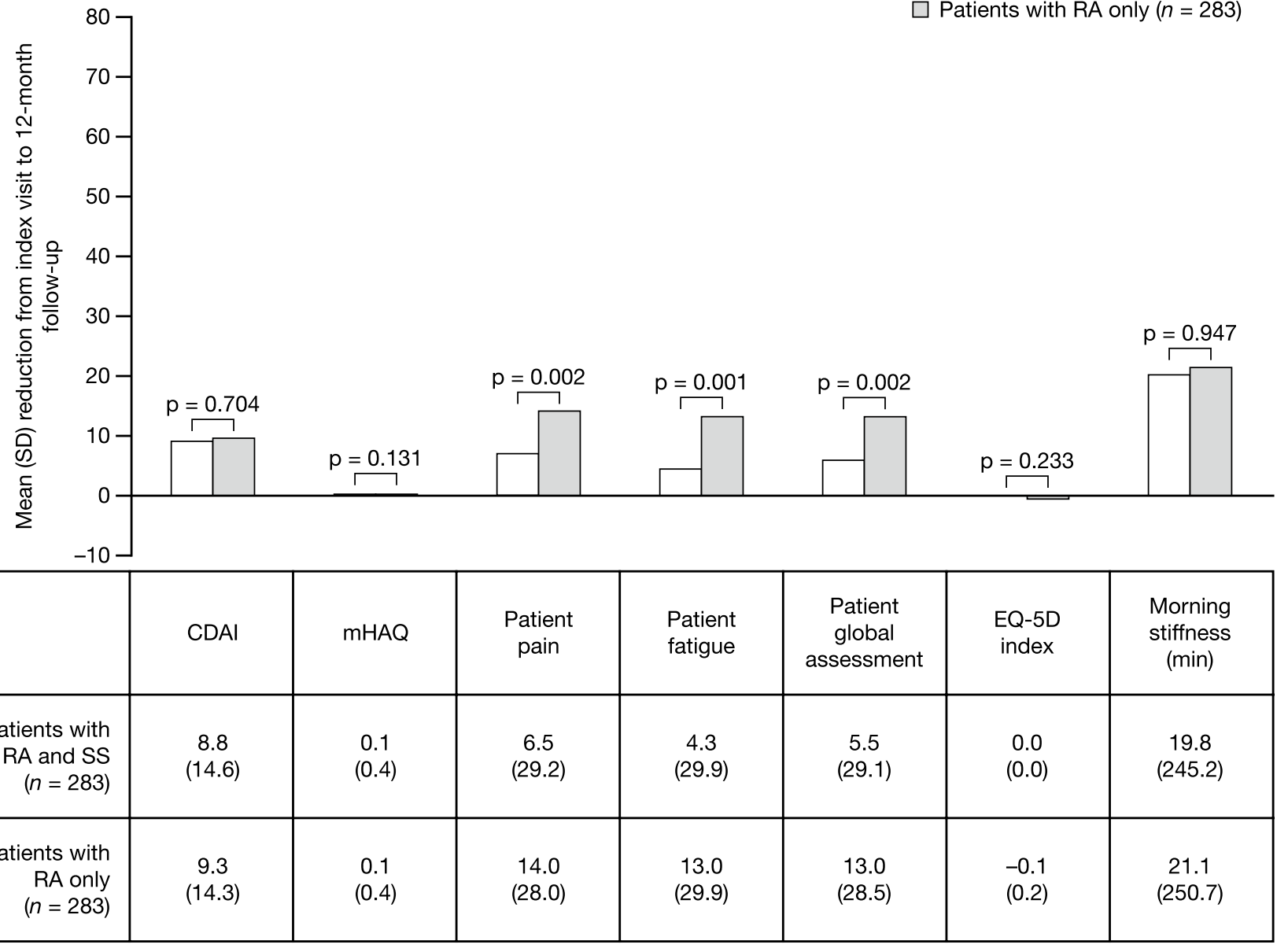

Fig. 2 Mean (SD) reduction in CDAI score and PROs from index visit to 12-month follow-up visit. $p$ values estimated using Student's $t$ test and Chi-square test. CDAI Clinical Disease Activity Index, EQ-

mean change in mHAQ was the same in both cohorts; there was no change in EQ-5D index score from index event until 12 month follow-up for patients with RA only, while the mean change for patients with RA and SS was marginally worse at 12 months.

\section{Discussion}

In this analysis of data from the large US Corrona RA registry, we compared RA disease activity and PROs among patients with RA, with and without SS, who initiated b/ tsDMARD treatment. Initially, patients with RA and SS or RA only were frequency matched by duration of RA. Significantly fewer patients with RA and SS were in full-time employment and more were disabled or retired compared with patients with RA only. Patients with RA and SS were also more likely to have co-morbidities and to be taking several treatments for RA. These results complement published
5D EuroQol 5-dimension, $m H A Q$ modified Health Assessment Questionnaire, $P R O$ patient-reported outcome, $R A$ rheumatoid arthritis, $S D$ standard deviation, SS Sjögren's syndrome

data examining the effect of SS on quality of life [8, 19]. The symptoms of SS exert a burden on a patient that may impact physical and social functioning, which in turn may affect employment status. Combined with the burden of RA [20], SS may substantially affect a patient's quality of life.

There is a paucity of published data investigating disease activity in patients with RA and SS, particularly from studies measuring cumulative disease activity with index dates defined by SS onset $[6,9,15,21-23]$; only two of the aforementioned studies included a follow-up period [6, 9]. The studies vary in size (82-1471 patients) $[9,22]$ and methods used for SS diagnosis, often utilizing a mixture of objective (e.g., Schirmer's test) and subjective methods (e.g., physician exam and/or questionnaire from patientreported symptoms) [21-23]. Two cross-sectional observational studies found no relationship between SS status and RA disease activity, as measured by Disease Activity Score 28 (DAS28) using erythrocyte sedimentation rate or C-reactive protein, in patients with $\mathrm{RA}$ with and 


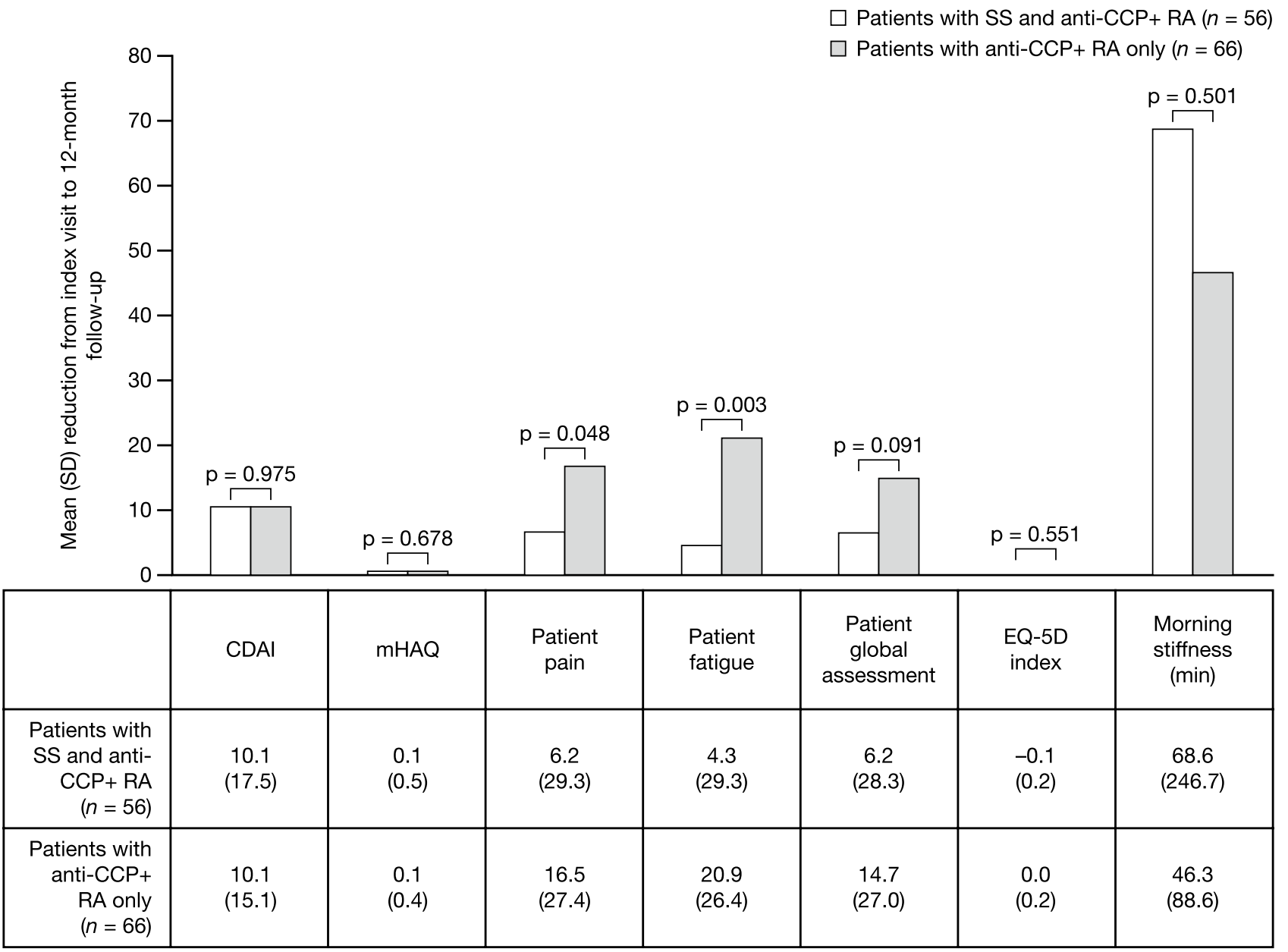

Fig. 3 Mean (SD) reduction in CDAI score and PROs from index visit to 12-month follow-up visit in the subgroup of patients with anti-CCP+RA. $p$ values estimated using Student's $t$ test and Chisquare test. $C C P+$ cyclic citrullinated peptide positive, $C D A I$ Clinical

without SS [22, 23]. It should be noted that the proportion of patients with RA and SS in these studies was small: $n=20 / 82$ [22] and $n=11 / 307$ [23]. In contrast, several studies have noted an association between SS and RA disease activity $[9,15,21]$. The analysis of 1471 patients with RA enrolled in a longitudinal RA registry revealed that patients with RA and SS $(n=415)$ experienced a longer duration of disease, higher RA disease activity (measured by RA disease activity index, CDAI and DAS28) at baseline and significantly lower reduction in RA disease activity at 12 months than patients with RA only [9]. In a study of 636 patients with RA ( $n=232$ patients with SS symptoms), DAS28 was significantly higher for patients with moderate and severe symptoms compared with patients with no symptoms of SS [21]. Similarly, among 509 patients with RA ( $n=74$ patients with RA and SS), patients with RA and SS had significantly higher DAS28 scores than patients with RA only [15].
Disease Activity Index, mHAQ modified Health Assessment Questionnaire, $P R O$ patient-reported outcome, $R A$ rheumatoid arthritis, $S D$ standard deviation, SS Sjögren's syndrome

To minimize selection bias in the current study, patients were PSM to account for variables associated with treatment response. The resulting cohorts (patients with RA and SS and patients with RA only) were generally well balanced in terms of baseline characteristics. At the 12-month follow-up visit, there were no significant differences in the change in clinical disease activity between patients with RA and SS and those with RA only; however, change in CDAI score after 12 months was numerically lower (suggesting greater impairment) in patients with RA and SS compared to those with RA only. The lack of association between SS status and RA disease activity in the present study is likely due to PSM; characteristics that are commonly seen with SS, and that are associated with RA disease activity, were accounted for in this analysis, but may not have been in the aforementioned studies, which did not use PSM [9, 15, 21].

Previous studies have demonstrated that patients with SS only (as opposed to patients with SS co-existing with another 
autoimmune condition) experience pain and fatigue [24], as do patients with RA only [25]; therefore, it could reasonably be inferred that, when co-existing with RA, SS exacerbates these PROs. In this analysis, at the 12-month followup visit, improvements in the majority of RA-related PROs were reduced in patients with RA and SS versus patients with RA only (suggesting more impairment). In accordance with this, several studies have demonstrated the additional burden of SS on patients with RA [9, 21, 23]. In an observational study, patients with RA with moderate or severe SS symptoms had significantly worse scores for mHAQ, pain and fatigue compared with patients with RA with no SS symptoms [21]. In a longitudinal registry analysis, patients with RA and SS experienced significantly higher multidimensional (MD) HAQ fatigue scores at baseline compared with patients with RA only [9]. After 12 months, patients with RA and SS experienced numerically lower reductions in MDHAQ fatigue scores than patients with RA only [9]. In addition, an observational study demonstrated that patients with RA and SS experienced numerically greater pain (visual analog scale) scores compared with patients with RA only [23]. Interestingly in our analysis, the differences in two PRO measures, mHAQ and EQ-5D index, between patients with RA and SS and patients with RA only, were marginal. However, the mHAQ and EQ-5D index questionnaires are completed by patients and would likely be impacted by underlying RA disease status.

Patients with RA who are ACPA+ are more likely to have a more severe disease course than patients who are ACPA- [13]. A study of Greek patients with RA concluded that the presence of anti-CCP antibodies was associated with extra-articular manifestations, such as serositis and pulmonary fibrosis [14]. In several studies, the proportion of patients with anti-CCP antibodies has been found to be higher in patients with RA and SS compared with patients with RA only (67.6-77.8\% versus 59.4-71.9\%) [6, 9, 15]. Therefore, we sought to clarify if the presence of SS would exacerbate the disease course further in anti-CCP+ patients. Contrary to previous research $[6,9,15]$, in the current study, the proportion of patients with anti-CCP antibodies was lower in patients with RA and SS compared with patients with RA only ( $49.1 \%$ versus $55.5 \%$ ); however, it should be noted that not all patients had measures reported. RA disease activity and PRO results were similar in the subgroup of patients with RA who were anti-CCP+, indicating that SS status did not affect outcomes differently in this patient population.

The biologic pathways associated with the differences in patients with RA with, and without, dry eyes and mouth (SS) are not clear [26-28]. However, the results of the current study do suggest that physicians may wish to consider SS status in the management of patients with RA; in particular, there may be a need for closer monitoring and assessment of response to treatment. More aggressive treatment of SS with RA may be needed.

This analysis has several strengths, namely the Corrona registry is the largest disease registry in the US that collects data directly from both providers and patients at the time of a routine clinical encounter. This allowed for patients with RA and SS or RA only to be selected from a broad population, ensuring satisfactory pairing [29]. The Corrona registry does include a wide variety of rheumatology practices participating throughout the country (rural and urban areas, academic and private settings) and brings access to broad geographic locations and patients with diverse sociodemographic origins. Prior analyses compared Medicare patients with RA enrolled in Corrona to those who are not part of the registry and found similar demographic and co-morbidity characteristics, supporting the generalizability of the Corrona registry [29]. Data are collected at regular intervals, which enabled us to evaluate outcomes at two different time points. Advanced epidemiological methods (e.g., PSM) were used to compare responses between patients with and without SS where selection bias may have existed. The presence of SS was captured on the provider form and is considered a critical field of data for Corrona. The results from this observational study in US patients complement previous studies $[8,9,19,21-23]$.

As opposed to previously reported studies, bias was minimized by PSM of the two cohorts (RA and SS versus RA only) by factors known to be associated with treatment response (e.g., age, sex, CDAI score, number of prior biologics, work status, history of co-morbidities and RA disease characteristics). Another limiting factor was that patients included in the registry were diagnosed by different rheumatologists across the US; diagnosis of SS was at the discretion of the treating physician (SS associated with RA: yes/no) and rheumatologists may have used a variety of mostly historical clinical signs and symptoms rather than objective testing (i.e., Schirmer's test). Nevertheless, we believe that the manner in which these data were collected reflects real-world clinical practice with the reporting of dry eyes and/or mouth not associated with medications or mouth breathing during the night. Additionally, patients in the RA-only cohort also required a questionnaire entry for SS associated with RA (yes/no), potentially leading to under-ascertainment.

In this large US patient population initiating b/tsDMARD treatment, patients with RA only had greater improvements in RA disease activity and PROs than those with RA and SS; similar results were shown in anti-CCP+ patients. Physicians may wish to consider SS status when managing patients with RA; specifically, patients with RA and SS may require closer monitoring and more aggressive intervention to improve their disease experience than patients with RA only. Additional studies are needed to further understand the 
biological pathways involved in SS co-existing with RA, and subsequently to provide targeted treatment options for this population of patients.

Acknowledgements The findings of this research were presented in poster format at the 2019 European League Against Rheumatism (EULAR) annual meeting: Harrold LR, Shan Y, Rebello S, et al. THU0110 Disease Activity and Patient-Reported Outcomes in Patients with RA with Sjögren's Syndrome Enrolled in a Large Observational US Registry. Annals of the Rheumatic Diseases 2019;78(Supp 2):327328 (https://doi.org/10.1136/annrheumdis-2019-eular.1957). The authors would like to thank all of the patients and providers who have participated in the Corrona RA registry. Corrona has been supported through contracted subscriptions in the last 2 years by AbbVie, Amgen, Boehringer Ingelheim, Bristol-Myers Squibb Company, Celgene, Crescendo, Eli Lilly and Company, Genentech, Gilead, GlaxoSmithKline, Janssen, Merck, Momenta Pharmaceuticals, Novartis, Ortho Dermatologics, Pfizer Inc., Regeneron, Roche, Sun, and UCB. The design and study conduct were a collaborative effort between Corrona and BristolMyers Squibb Company, and financial support for the study was provided by Bristol-Myers Squibb Company. Professional medical writing support and editorial assistance was provided by Rachel Rankin, $\mathrm{PhD}$ at Caudex, and was funded by Bristol-Myers Squibb Company.

Author contributions All authors made substantial contributions to the conception and design of the study, or acquisition of data, or analysis and interpretation of data; drafted the article or revised it critically for important intellectual content; gave final approval of the version to be submitted; and agreed to be accountable for all aspects of the work in ensuring that questions related to the accuracy or integrity of any part of the work are appropriately investigated and resolved.

Funding This study was sponsored by Corrona, LLC and funded by Bristol-Myers Squibb Company. Professional medical writing support and editorial assistance was funded by Bristol-Myers Squibb Company.

Availability of data and material The datasets generated and/or analyzed during the current study are not publicly available, but are available from the corresponding author upon reasonable request. The Corrona dataset is based on a large US multicenter study adhering to a number of institutional review boards, with complex logistics. Patients did not provide consent to raw data sharing during the data collection for this purpose, and the Corrona data-sharing policies do not permit raw data sharing for this purpose. An aggregated limited dataset from the current analyses is available to qualified investigators with an approved protocol. Data requests may be sent to Corrona, represented by Dr. Jeffrey D. Greenberg MD MPH, NYU School of Medicine, New York, NY, e-mail jgreenberg@ corrona.org.

Code availability Not applicable.

\section{Compliance with ethical standards}

Conflict of interest Leslie R. Harrold is an employee of Corrona, LLC, a shareholder in Corrona, LLC and has received grant/research support from Pfizer and consulting fees from AbbVie, Bristol Myers Squibb, and Roche. Corrona, LLC has received funds from Bristol Myers Squibb for the analyses. Ying Shan has nothing to disclose. Sabrina Rebello is an employee of Corrona, LLC. Neil Kramer owns stock in AbbVie, Amgen, Johnson \& Johnson, Pfizer and Regeneron. Evo Alemao is an employee of Bristol Myers Squibb. Sean E. Connolly and Sheila Kelly have stock options/bond holdings in, and are employees of, Bristol Myers Squibb. Joel Kremer has stock options/bond holdings in, and is an employee of, Corrona, LLC, has received consultancy fees from AbbVie, Amgen, Bristol Myers Squibb, Genentech, Lilly, Regeneron, Sanofi, and Pfizer; grant/research support from AbbVie, Bristol Myers Squibb, Genentech, Lilly, Novartis and Pfizer. Elliot D. Rosenstein reports serving on an advisory board and speakers bureau for Bristol Myers Squibb.

Ethics approval The database used in this study was the Corrona RA registry. This study was carried out in accordance with the Declaration of Helsinki. All participating investigators were required to obtain full institutional review board approval for conducting non-interventional research involving human subjects. Sponsor approval and continuing review was obtained through a central Institutional Review Board (IRB; New England Independent Review Board, NEIRB No. 120160610). For academic investigative sites that did not receive a waiver to use the central IRB, full board approval was obtained from the respective governing IRBs and documentation of approval was submitted to Corrona, LLC prior to initiating any study procedures. All registry patients were required to provide written informed consent and authorization prior to participating.

Informed consent Consent to participate: All registry patients were required to provide written informed consent and authorization prior to participating. Consent for publication: Not applicable.

Open Access This article is licensed under a Creative Commons Attribution 4.0 International License, which permits use, sharing, adaptation, distribution and reproduction in any medium or format, as long as you give appropriate credit to the original author(s) and the source, provide a link to the Creative Commons licence, and indicate if changes were made. The images or other third party material in this article are included in the article's Creative Commons licence, unless indicated otherwise in a credit line to the material. If material is not included in the article's Creative Commons licence and your intended use is not permitted by statutory regulation or exceeds the permitted use, you will need to obtain permission directly from the copyright holder. To view a copy of this licence, visit http://creativecommons.org/licenses/by/4.0/.

\section{References}

1. Patel R, Shahane A (2014) The epidemiology of Sjogren's syndrome. Clin Epidemiol 6:247-255. https://doi.org/10.2147/CLEP. S47399

2. Mavragani CP, Moutsopoulos HM (2019) Primary versus secondary Sjogren syndrome: is it time to reconsider these terms? J Rheumatol 46:665-666. https://doi.org/10.3899/jrheum.180392

3. Singh JA, Saag KG, Bridges SL et al (2016) 2015 American College of Rheumatology guideline for the treatment of rheumatoid arthritis. Arthritis Care Res 68:1-26. https://doi.org/10.1002/ art.39480

4. Smolen JS, Landewé R, Bijlsma J et al (2017) EULAR recommendations for the management of rheumatoid arthritis with synthetic and biological disease-modifying antirheumatic drugs: 2016 update. Ann Rheum Dis 76:960-977. https://doi.org/10.1136/ annrheumdis-2016-210715

5. Shiboski CH, Shiboski SC, Seror R et al (2017) 2016 American College of Rheumatology/European League Against Rheumatism classification criteria for primary Sjogren's syndrome: a consensus and data-driven methodology involving three international patient cohorts. Ann Rheum Dis 76:9-16. https://doi.org/10.1136/annrh eumdis-2016-210571 
6. Brown LE, Frits ML, Iannaccone CK et al (2015) Clinical characteristics of RA patients with secondary SS and association with joint damage. Rheumatology (Oxford) 54:816-820. https://doi. org/10.1093/rheumatology/keu400

7. Alemao E, Saini, Y, Bao, Y et al (2018) Do anti-citrullinated protein antibodies and anti-Sjögren's-syndrome-related antigen a double positive patients with secondary Sjögren's syndrome and RA have higher joint disease activity? [abstract]. Arthritis Rheumatol 70(suppl 10):abstract 1463

8. Mertzanis P, Abetz L, Rajagopalan K et al (2005) The relative burden of dry eye in patients' lives: comparisons to a U.S. normative sample. Investig Ophthalmol Vis Sci 46:46-50. https://doi. org/10.1167/iovs.03-0915

9. Alemao E, Saini, Y, Bao Y et al (2018) The joint disease burden in patients with secondary Sjögren's syndrome and RA compared to patients with RA only [abstract]. Arthritis Rheumatol 70(suppl 10):abstract 2506

10. Ware JE, Snow KK, Kosinski M et al (1993) SF-36 health survey: manual and interpretation guide. The Health Institute, New England Medical Center, Boston

11. Payet J, Belkhir R, Gottenberg JE et al (2015) ACPA-positive primary Sjogren's syndrome: true primary or rheumatoid arthritisassociated Sjogren's syndrome? RMD Open 1:e000066. https:// doi.org/10.1136/rmdopen-2015-000066

12. Kurowska W, Kuca-Warnawin EH, Radzikowska A et al (2017) The role of anti-citrullinated protein antibodies (ACPA) in the pathogenesis of rheumatoid arthritis. Cent Eur J Immunol 42:390398. https://doi.org/10.5114/ceji.2017.72807

13. van der Helm-van Mil AHM, Verpoort KN, Breedveld FC et al (2005) Antibodies to citrullinated proteins and differences in clinical progression of rheumatoid arthritis. Arthritis Res Ther 7:R949-958. https://doi.org/10.1186/ar1767

14. Alexiou I, Germenis A, Koutroumpas A et al (2008) Anti-cyclic citrullinated peptide-2 (CCP2) autoantibodies and extra-articular manifestations in Greek patients with rheumatoid arthritis. Clin Rheumatol 27:511-513. https://doi.org/10.1007/s1006 7-007-0800-1

15. He J, Ding Y, Feng M et al (2013) Characteristics of Sjogren's syndrome in rheumatoid arthritis. Rheumatology (Oxford) 52:1084-1089. https://doi.org/10.1093/rheumatology/kes374

16. Kremer JM (2016) The Corrona US registry of rheumatic and autoimmune diseases. Clin Exp Rheumatol 34:S96-S99

17. Austin PC (2011) An introduction to propensity score methods for reducing the effects of confounding in observational studies. Multivariate Behav Res 46:399-424. https://doi.org/10.1080/00273 171.2011.568786

18. Aletaha D, Smolen J (2005) The Simplified Disease Activity Index (SDAI) and the Clinical Disease Activity Index (CDAI): a review of their usefulness and validity in rheumatoid arthritis. Clin Exp Rheumatol 23:S100-108

19. Omma A, Tecer D, Kucuksahin O et al (2018) Do the European League Against Rheumatism (EULAR) Sjogren's syndrome outcome measures correlate with impaired quality of life, fatigue, anxiety and depression in primary Sjogren's syndrome? Arch Med Sci 14:830-837. https://doi.org/10.5114/aoms.2017.70300

20. Ferro F, Elefante E, Luciano N et al (2017) One year in review 2017: novelties in the treatment of rheumatoid arthritis. Clin Exp Rheumatol 35:721-734

21. Uhlig T, Kvien TK, Jensen JL et al (1999) Sicca symptoms, saliva and tear production, and disease variables in 636 patients with rheumatoid arthritis. Ann Rheum Dis 58:415-422. https://doi. org/10.1136/ard.58.7.415

22. Antero DC, Parra AG, Miyazaki FH et al (2011) Secondary Sjogren's syndrome and disease activity of rheumatoid arthritis. Rev Assoc Med Bras 57:319-322

23. Haga HJ, Naderi Y, Moreno AM et al (2012) A study of the prevalence of sicca symptoms and secondary Sjogren's syndrome in patients with rheumatoid arthritis, and its association to disease activity and treatment profile. Int J Rheum Dis 15:284-288. https ://doi.org/10.1111/j.1756-185X.2012.01717.x

24. Lackner A, Ficjan A, Stradner MH et al (2017) It's more than dryness and fatigue: the patient perspective on health-related quality of life in Primary Sjogren's Syndrome-a qualitative study. PLoS ONE 12:e0172056. https://doi.org/10.1371/journal.pone.0172056

25. McWilliams DF, Walsh DA (2017) Pain mechanisms in rheumatoid arthritis. Clin Exp Rheumatol 35(Suppl 107):94-101

26. Oliveira HF, de Souza TR, Carvalho CN et al (2015) Serologic profile and clinical markers of Sjogren syndrome in patients with rheumatoid arthritis. Oral Surg Oral Med Oral Pathol Oral Radiol 119:628-635. https://doi.org/10.1016/j.oooo.2015.02.479

27. Aluru SV, Shweta A, Bhaskar S et al (2017) Tear fluid protein changes in dry eye syndrome associated with rheumatoid arthritis: a proteomic approach. Ocul Surf 15:112-129. https://doi. org/10.1016/j.jtos.2016.09.005

28. Liao CC, Chou PL, Cheng CW et al (2016) Comparative analysis of novel autoantibody isotypes against citrullinated-inter-alphatrypsin inhibitor heavy chain 3 (ITIH3)(542-556) peptide in serum from Taiwanese females with rheumatoid arthritis, primary Sjogren's syndrome and secondary Sjogren's syndrome in rheumatoid arthritis. J Proteomics 141:1-11. https://doi.org/10.1016/j. jprot.2016.03.031

29. Curtis JR, Chen L, Bharat A et al (2014) Linkage of a de-identified United States rheumatoid arthritis registry with administrative data to facilitate comparative effectiveness research. Arthritis Care Res (Hoboken) 66:1790-1798. https://doi.org/10.1002/acr.22377

Publisher's Note Springer Nature remains neutral with regard to jurisdictional claims in published maps and institutional affiliations. 